\title{
Study of the deposition and characterisation of a 5,10,15,20-tetrakis-(4-sulphonatophenyl)porphyrin Co(II) layer at gold surfaces in alkaline solution
}

\author{
Karolien De Wael $^{\mathrm{a}, *, 1}$, Philippe Westbroek ${ }^{\mathrm{b}}$, Peter Vandenabeele ${ }^{\mathrm{c}, 2}$, \\ Annemie Adriaens ${ }^{\mathrm{a}}$, Eduard Temmerman ${ }^{\mathrm{a}}$ \\ ${ }^{a}$ Ghent University, Department of Analytical Chemistry, Krijgslaan 281 S12, B-9000 Ghent, Belgium \\ ${ }^{\mathrm{b}}$ Ghent University, Department of Textiles, Technologiepark 907, B-9052 Ghent, Belgium \\ ${ }^{c}$ Ghent University, Department of Analytical Chemistry, Proeftuinstraat 86, B-9000 Ghent, Belgium
}

Received 31 August 2004; received in revised form 19 February 2005; accepted 25 March 2005

\begin{abstract}
In this paper, the electrodeposition of Co(II)TSPor at gold electrodes is described. It was found that this deposition is initially controlled by kinetic parameters leading to a nearly $100 \%$ coverage of the electrode surface. However, once formed a reorganisation of the layer occurs. Experimental evidence that Co(II)TSPor is deposited was provided by using Raman spectroscopy.
\end{abstract}

(C) 2005 Elsevier Ltd. All rights reserved.

Keywords: Gold; Electrodeposition; Electrocatalysis; Porphyrins; Modification

\section{Introduction}

Porphyrins are widespread compounds in nature. Natural colours are often related to porphyrins. They are important for several mechanisms in life: they catalyse enzymatic reactions and are, for instance, responsible for the oxygen transport in the human body [1]. Phthalocyanines, frequently used molecules in electrocatalysis [2-5], are closely related to these naturally occurring porphyrins. The basic structure of porphyrin consists of four pyrrole units which are linked by four methine bridges. The porphyrin macrocycle is geometrically flat and has an conjugated $18 \pi$-electron system. Following Hückel's rule, the porphyrin ring can be considered as an aromatic system. The possibility of delocalization

\footnotetext{
* Corresponding author. Tel.: +32 926448 20; fax: +329 2644960 .

E-mail address: karolien.dewael@ugent.be (K.D. Wael).

${ }^{1}$ Research Assistant of the Fund for Scientific Research, Flanders (Belgium) (F.W.O., Vlaanderen).

${ }^{2}$ Postdoctoral Fellow of the Fund for Scientific Research, Flanders (Belgium) (F.W.O., Vlaanderen).
}

of the electrons is important for its chemical activity and biological functions. The porphyrin ring is very stable towards concentrated acids and bases. Its major characteristics are its thermal and chemical stability, coupled with its extensive redox chemistry.

The aromatic ring structure can be electrochemically oxidised and/or reduced. More than 70 different metal ions can form a complex by coordinating with the porphyrin ring. When the central metal is a transition metal, its ion can also be oxidised and/or reduced. The solubility of the porphyrin in an aqueous solution can be efficiently altered by substitution of sulfonatophenyl groups [6,7].

In this paper, the electrochemical behavior of 5,10,15,20tetrakis-(4-sulphonatophenyl) porphyrin Co(II), tetrasodium salt (CoTSPor, Fig. 1) at a gold electrode in alkaline solution is described. The immobilization of CoTSPor on a gold electrode can lead to a modified electrode with electrocatalytic properties [8]. Cobalt(II) was selected based on its well-known redox behaviour and electrocatalytic properties.

Electrodeposition is the method used in this study to deposit a film of CoTSPor onto a gold electrode. A continuous 


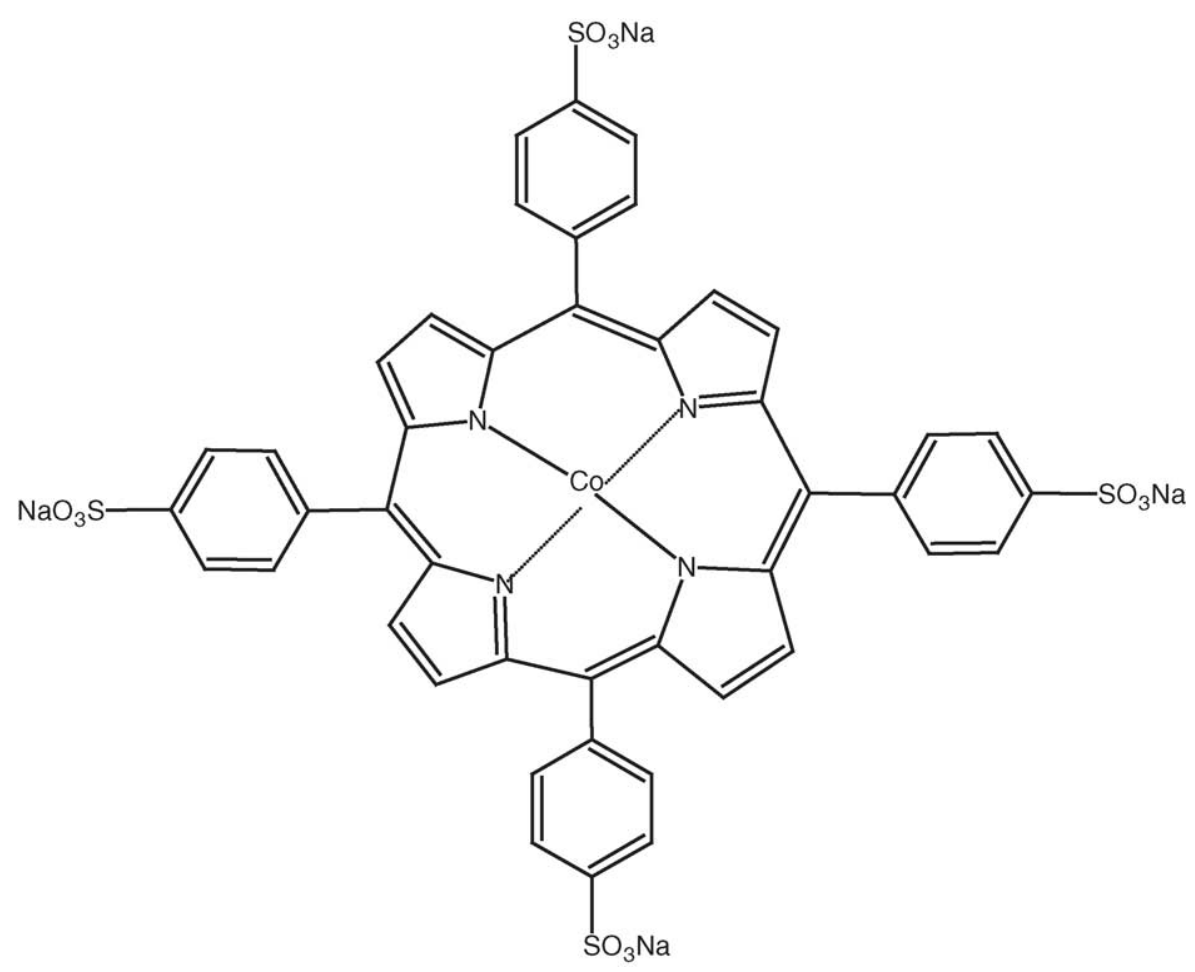

Fig. 1. Molecular structure of 5,10,15,20-tetrakis-(4-sulfonatophenyl)porphyrin cobalt(II), tetrasodium salt.

potential cycling of the electrode between two potentials in a CoTSPor solution can lead to a modification of the bare electrode. By measuring the current, it is possible to follow the adsorption process in situ.

Characterisation of the modified electrodes is done by electrochemical and spectroscopic methods, including Raman spectroscopy.

\section{Experimental}

A saturated calomel reference electrode (SCE) with two compartments (radiometer) and a carbon counter electrode were used. The working electrodes were gold electrodes with a diameter of $1.6 \mathrm{~mm}$ (purity $99.95 \%$, BAS, England) and were pretreated by mechanical and electrochemical polishing. Before its first use, the electrode surface was scoured briefly on SiC-emery paper 1200 grit to obtain a fresh surface. To smoothen this relatively rough surface, it was further subjected to sequential polishing on a polishing cloth covered with alumina (Buehler) powder of $1,0.3$ and $0.05 \mu \mathrm{m}$ particle size for respectively 5,10 and $20 \mathrm{~min}$. To remove any adherent $\mathrm{Al}_{2} \mathrm{O}_{3}$ particles, the electrode surface was rinsed thoroughly with doubly deionised water and cleaned in an ultrasonic bath (Branson 3210) for $2 \mathrm{~min}$. Finally, the electrode was pretreated electrochemically by

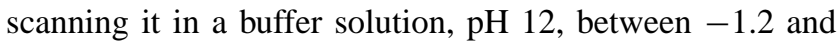
$0.6 \mathrm{~V}$ versus SCE until five subsequent scans were identical. The scan rate for all cyclic voltammetric experiments was $50 \mathrm{mV} \mathrm{s}^{-1}$.
A PGSTAT20 potentiostat (ECO Chemie, the Netherlands) controlled by GPES 4.9 software package running on a Pentium II computer (Eknadata) was used to record the voltammetric curves. Measurement of the $\mathrm{pH}$ of the solution was done with a Orion Benchtop pH-meter model 420A. The CoTSPor sodium salt (purity 98\%) was purchased from Porphyrin Systems (Lübeck, Germany). The buffer solution of $\mathrm{pH} 12\left(\mathrm{Na}_{2} \mathrm{HPO}_{4} / \mathrm{NaOH}\right)$ and sodium dithionite were purchased from Riedel-de Haën (Belgium). Before each experiment, pure nitrogen was bubbled through the cell solution for $20 \mathrm{~min}$.

Raman analysis of the modified electrodes has been performed on a Rensihaw system- 1000 spectrometer (Renishaw, Wotton Under Edge, UK). Electrodes were mounted on the microscope table and the laser light $(780 \mathrm{~nm}$, ca. $5 \mathrm{~mW}$ at the sample) was focussed directly on the electrode surface by using an Olympus 50X objective, obtaining a spot size of ca. $2 \mu \mathrm{m}$ diameter. The backscattered radiation was collected by the same objective lens and the Rayleigh line was suppressed by a notch filter. The light was dispersed over a 1200 lines/mm diffraction grating and was collected on a Peltier cooled charge coupled device (CCD) detector. Spectra were recorded in the Stokes branch of the spectrum, between 300 and $1700 \mathrm{~cm}^{-1}$.

\section{Results and discussion}

The first 19 cyclic voltammetric scans recorded at a gold electrode in a pH 12 buffer solution (curve 1) and a 

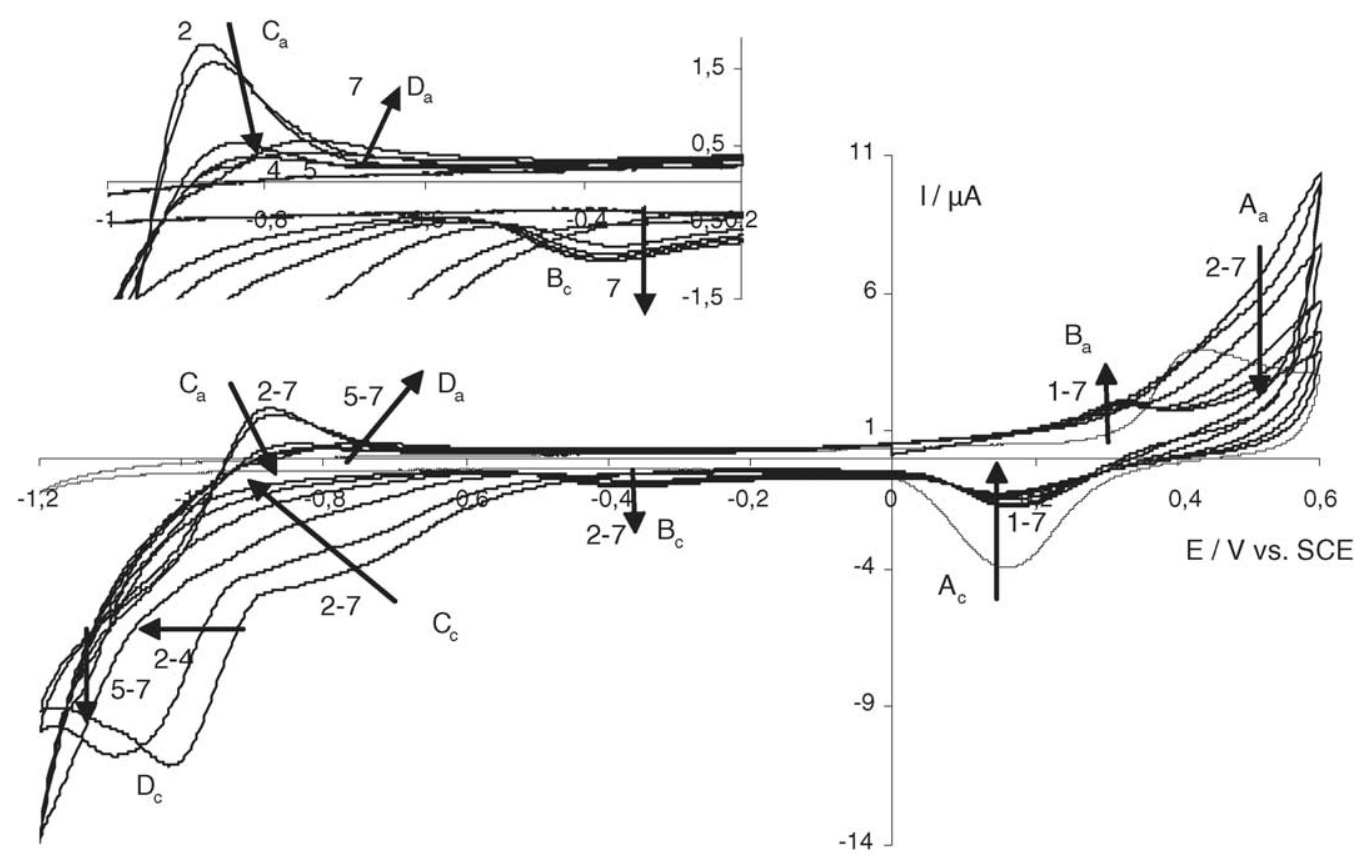

Fig. 2. Current-potential curves recorded at a gold disc electrode in a pH 12 buffer solution in the absence (curve 1, dotted line) and presence (curves 2-7) of $8.04 \times 10^{-3} \mathrm{~mol} \mathrm{~L}^{-1} \mathrm{Co}(\mathrm{II}) \mathrm{TSP}$ r at a scan rate of $50 \mathrm{mV} \mathrm{s}^{-1}$ and a temperature of $298.0 \mathrm{~K}$ as a function of scan number. Scan numbers are: (2) 1 ; (3) 2 ; (4) 6 ; (5) 10 ; (6) 14 and (7) 19. Inset: enlarged view of the -1 to $-0.2 \mathrm{~V}$ potential region.

buffer solution containing $8.04 \times 10^{-3} \mathrm{~mol} \mathrm{~L}^{-1} \mathrm{Co}(\mathrm{II}) \mathrm{TSP}$ or (curves 2-7) are shown in Fig. 2. The voltammetric behaviour is similar, but not identical, to the behaviour of a gold electrode in a CoTSPc (cobalt(II) tetrasulphonated phthalocyanine sodium salt) solution [9]. In what follows, the differences between both molecules concerning their electrochemistry will be explained. The peaks, corresponding to the oxidation of the gold surface $\left(\mathrm{A}_{\mathrm{a}}\right)$ and the reduction of the gold oxide formed during surface oxidation $\left(\mathrm{A}_{\mathrm{c}}\right)$, decrease markedly during the first 19 scans when cycled in a CoTSPor solution. This is a first indication of adsorption of CoTSPor onto the gold electrode, as covering the gold surface with CoTSPor prevents its oxidation and reduction.

In curves $2-7$, it can be seen that three new oxidation peaks, at about $0.3 \mathrm{~V}\left(\mathrm{~B}_{\mathrm{a}}\right),-0.9 \mathrm{~V}\left(\mathrm{C}_{\mathrm{a}}\right)$ and $-0.8 \mathrm{~V}\left(\mathrm{D}_{\mathrm{a}}\right)$ versus SCE, and three new reduction waves, at about $-0.4 \mathrm{~V}$ $\left(\mathrm{B}_{\mathrm{c}}\right),-0.8 \mathrm{~V}\left(\mathrm{C}_{\mathrm{c}}\right)$ and $-1 \mathrm{~V}\left(\mathrm{D}_{\mathrm{c}}\right)$ versus SCE, occur. The mentioned values of the peak potential of processes $C_{c}$ and $D_{c}$ are only valid for the first scan because their peak maximum shifts towards more negative potentials with increasing scan number. The decrease of oxidation peak $\mathrm{C}_{\mathrm{a}}$ and the increase of oxidation peak $B_{a}$ and reduction peaks $B_{c}$ and $C_{c}$ continue until scan 19. Process $D_{a}$ appears from scan 10 and its peak height increases from scan 10 until scan 19 . The peak maximum of $D_{c}$ shifts towards more negative potentials in the first 10 scans, which suggests that the completed shift of peak potential of peak $D_{c}$ initiates the appearance of peak $D_{\mathrm{a}}$. Continuation of the scanning beyond scan 19 results in a different behaviour (Fig. 3).

Fig. 3 shows curves obtained after scan 19, from scan 19 (curve 1) to scan 100 (curve 4). It is remarkable that oxidation

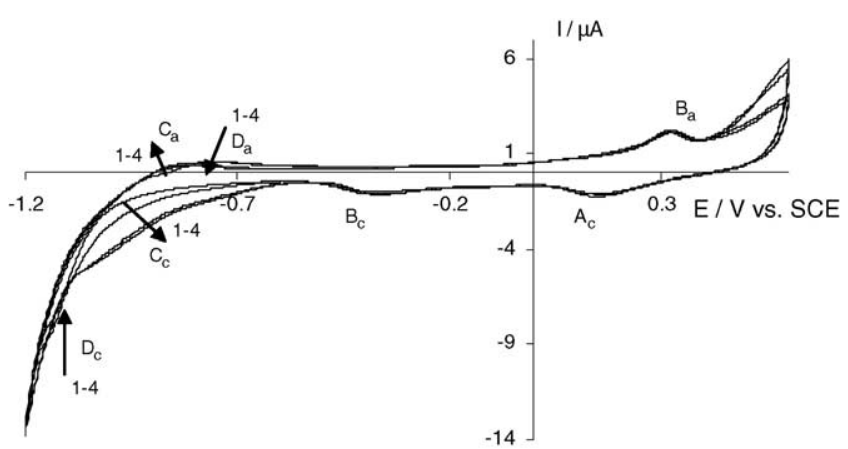

Fig. 3. Current-potential curves recorded at a gold disc electrode in a $\mathrm{pH} 12$ buffer solution containing $8.04 \times 10^{-3} \mathrm{~mol} \mathrm{~L}^{-1} \mathrm{Co}$ (II)TSPor at a scan rate of $50 \mathrm{mV} \mathrm{s}^{-1}$ and a temperature of $298.0 \mathrm{~K}$ as a function of scan number. Scan numbers are: (1) 19; (2) 50; (3) 80 and (4) 100.

peak $B_{a}$ and both reduction peaks $B_{c}$ and $A_{c}$ stabilise after scan 19. Processes $C$ and $D$ reverse in their increasing or declining behaviour. After scan 19, peak currents of $C_{a}$ and $C_{c}$ increase, while the peaks of wave $D_{a}$ and $D_{c}$ decrease. Similar to the CoTSPc study [9], scan 19 can be considered as the scan of maximum coverage. Before scan 19 , peaks $C_{a}$ and $\mathrm{C}_{\mathrm{c}}$, as well as processes $\mathrm{D}_{\mathrm{a}}$ and $\mathrm{D}_{\mathrm{c}}$, can be attributed to the ring reduction and oxidation of CoTSPor in solution.

It is also important to note that the rate of decrease of peak $\mathrm{A}_{\mathrm{c}}$ is different from the rate of increase of process $\mathrm{B}_{\mathrm{c}}$. Fig. 4 shows the relationship between the charge of processes $A_{c}$ and $B_{c}$ and the scan number. Two different slopes $\left(A_{c}\right)$ indicates the convolution of two processes, one increasing in time (adsorption), one decreasing in time (gold oxide reduction). This is rather clear because process $A_{c}$ is the result of more 


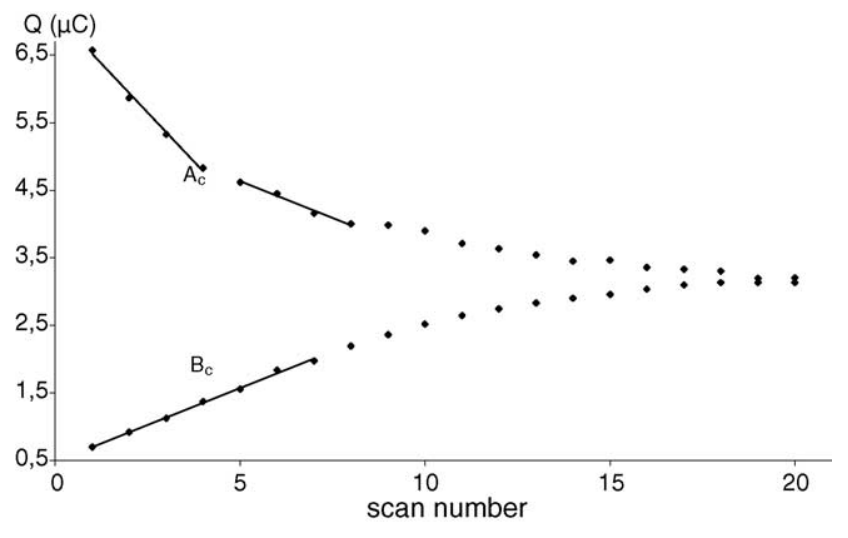

Fig. 4. Relationship between charge of processes $B_{c}$ and $A_{c}$ and scan number.

than one reaction. The peak current decreases due to covering of the gold surface and increases due to electroactive properties of deposited $\mathrm{Co}$ (II)TSPor at the same potential (ring reduction of adsorbed CoTSPor).

For scan 19, the charge related to oxidation wave $B_{a}$ and the charge related to the reduction waves $B_{c}$ and $A_{c}$ is almost the same and equal to $3.2 \pm 0.5 \mu \mathrm{C}$. Fig. 4 shows that the charges of $B_{c}$ and $A_{c}$ are equal. Therefore, it is supposed that process $A_{c}$ after scan 19 is only due to the reduction of the adsorbed fraction of CoTSPor, which means that at scan 19, a maximum value for the surface coverage is obtained. No more gold can be oxidized $\left(\mathrm{A}_{\mathrm{a}}\right)$ or no more gold oxides can be reduced $\left(\mathrm{A}_{\mathrm{c}}\right)$. At the potential of process, $\mathrm{A}_{\mathrm{c}}$ only the ring reduction of adsorbed CoTSPor takes place at scan 19 . The increase with scan number of peaks $B_{a}$ and $B_{c}$ is due to the deposition of $\mathrm{Co}$ (II)TSPor at the gold surface that grows with scan number until scan 19 . Process A is, before scan 19, related to the gold reaction and a CoTSPor adsorption reaction. After scan 19, process A can be explained as an adsorption reaction of CoTSPor. No gold related processes occur because at scan 19 all gold is covered with CoTSPor.

In correlation with the data found in literature [9-11], peaks $B_{a}$ and $B_{c}$ are attributed to the Co(III)TSPor ${ }_{\text {ads }} /$ $\mathrm{Co}$ (II)TSPor ${ }_{\mathrm{ads}}$ redox system. Fig. 5 represents the voltammetric behaviour of a CoTSPor modified electrode in a $\mathrm{pH}$ 12 buffer solution. Only the adsorption processes are now

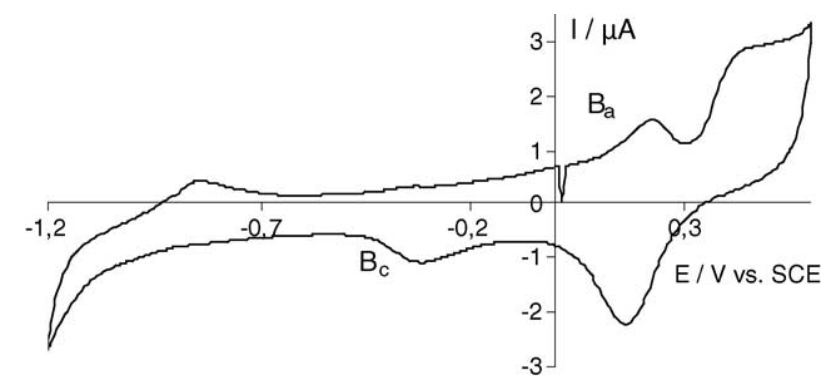

Fig. 5. Current-potential curve of scan 2 of a continuous cyclic voltammetric scanning experiment recorded at a gold electrode modified with CoTSPor in a $\mathrm{pH} 12$ buffer solution. present in the figure. Processes $\mathrm{B}_{\mathrm{c}}$ and $\mathrm{B}_{\mathrm{a}}$ stand for the reduction and oxidation of the $\mathrm{Co}(\mathrm{III})_{\mathrm{ads}} / \mathrm{Co}(\mathrm{II})_{\mathrm{ads}}$ redox system. Because of the considerable difference in peak potential, this process can be classified as irreversible. The charge under the peaks for these processes as well as the charge associated with peak $A_{c}$ is equal to $3.2 \pm 0.5 \mu \mathrm{C}$. Knowing that the charge of one electron is $1.6 \times 10^{-19} \mathrm{C}$ it follows that about $2.0 \times 10^{13}$ electrons are exchanged in each reaction. This leads to the conclusion that $0.97 \times 10^{15} \mathrm{~mol} \mathrm{~L}^{-1} \mathrm{CoT}$ SPor or $1.61 \times 10^{-9} \mathrm{~mol} \mathrm{~L}^{-1}$ CoTSPor is deposited on $1 \mathrm{~cm}^{2}$ of gold surface. Supposing the formation of a monolayer, each CoTSPor molecule would cover $0.1 \mathrm{~nm}^{2}$. Nevertheless, a CoTSPor molecule deposited in a flat configuration is expected to cover about $2 \mathrm{~nm}^{2}$. This means that Co(II)TSPor is adsorbed perpendicularly and/or a multilayer is formed. However, the changes in the peaks as a function of scan number observed in Figs. 2 and 3 indicate that a mixture of flat and perpendicular orientation of the molecules is obtained. The process before scan 19 is kinetically controlled, explaining the chaotic orientation of the CoTSPor molecules. In the CoTSPc study, it was seen that the situation changed considerably after the maximum coverage was obtained. In the case of CoTSPor, only small differences are observed after scan 19 (Fig. 3). The changes observed after the maximum coverage was reached in the case of CoTSPc were explained as the reorganisation of the deposited layer to form, from a thermodynamic point of view, a more stable layer consisting of columnar aggregates [8]. The CoTSPor molecules are not able to create such columnar aggregates because of the free benzyl groups on the porphyrin ring. These groups are perpendicularly oriented on the ring, which prevents the CoTSPor molecules to stick together and explains the slow evolution after that the maximum coverage was obtained.

Finally, processes $D_{c}$ and $D_{a}$ can be explained as the catalytic ring reduction and oxidation of CoTSPor in solution on a chaotic multilayer of CoTSPor molecules. Processes $\mathrm{C}_{\mathrm{c}}$ and $\mathrm{C}_{\mathrm{a}}$ can be interpreted as the ring reduction and oxidation on a less chaotic oriented CoTSPor multilayer. Electrochemical measurements of TSPor without Co also show a reduction wave at these potentials. The slow evolution of these last two peaks after the breaking point can be explained by the disability of the CoTSPor molecules to form a closely packed multilayer.

\subsection{Scan rate study}

The influence of the scan rate $(v)$ on the peak current $\left(I_{\mathrm{p}}\right)$ is investigated to find out whether the reactions attributed to the peaks observed in Figs. 2 and 3 are due to adsorption or to a diffusion controlled process. A slope of the relationship between $\log I_{\mathrm{p}}$ and $\log v$ equal to 1 would correspond to oxidation or reduction of CoTSPor adsorbed at the gold electrode, while a slope of 0.5 rather indicates a rate determining diffusion of CoTSPor or another species, such as hydroxide ions, towards the electrode surface [12-14]. Fig. 6 shows the relationship between $\log I_{\mathrm{p}}$ and $\log v$ for the different peaks 


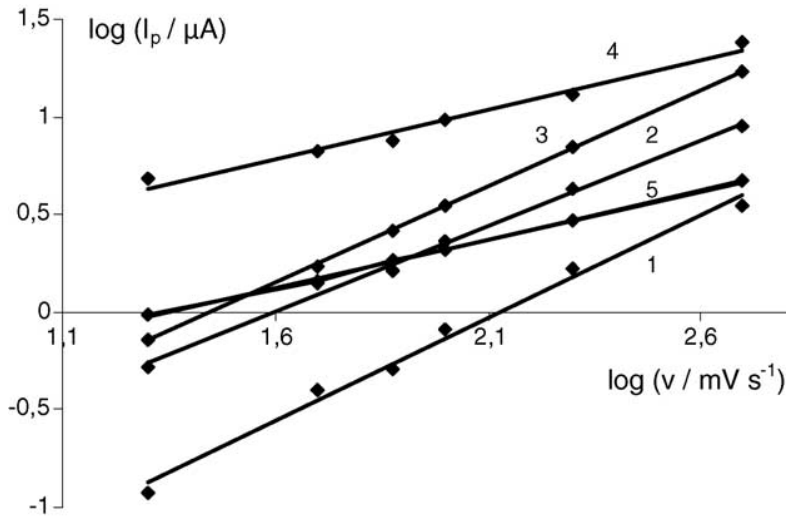

Fig. 6. Logarithmic plot of the peak current of the peaks observed in Figs. 2 and 3 as a function of scan rate. Curve: (1) peak $B_{c}$, (2) peak $A_{c}$, (3) peak $B_{a}$, (4) peak $D_{c}$ and (5) peak $D_{a}$.

observed. Peaks $D_{a}$ and $D_{c}$ can be considered as resulting from diffusion controlled reactions because of the $\log I_{\mathrm{p}}$ versus $\log v$ slope value of 0.5 (0.494 and 0.502). Variation of the CoTSPor concentration, while the concentration of the other compounds in solution was kept constant, showed that these peaks are due to diffusion of Co(II)TSPor towards the electrode surface and not to another compound because of the linear relationshiop obtained between peak current and $\mathrm{Co}$ (II)TSPor concentration. The other processes are due to adsorption reactions with a value of 1 for the $\log I_{\mathrm{p}}$ versus $\log v$ slope $\left(\mathrm{B}_{\mathrm{c}}, 1.048 ; \mathrm{A}_{\mathrm{c}}, 0.870\right.$ and $\left.\mathrm{B}_{\mathrm{a}}, 0.987\right)$.

\subsection{Concentration study}

Similar results were obtained when the same experiments as above were done for other CoTSPor concentrations. The same peaks were observed, only the scan corresponding with a maximum coverage was different for each concentration. A concentration of $8.04 \mathrm{mmol} \mathrm{L}^{-1}$ CoTSPor needs 19 scans to reach the break in the trend of the behaviour, corresponding to a maximum coverage of the electrode surface. A concentration of $5.92 \mathrm{mmol} \mathrm{L}^{-1}$ CoTSPor demands 42 scans, $3.95 \mathrm{mmol} \mathrm{L}^{-1}$ CoTSPor demands 59 scans and $1.97 \mathrm{mmol} \mathrm{L}^{-1}$ CoTSPor demands 82 scans. The smaller the concentration of CoTSPor, the longer it takes to reach the point of maximum coverage. This is expected because more CoTSPor in solution means more of it is transported towards the electrode surface per time unit. The relationship between the number of scans to reach the maximum coverage and the concentration CoTSPor is linear.

\subsection{Characterisation of the CoTSPor layer}

Fig. 6 already provides evidence of the occurrence of adsorption associated with several peaks based on electrochemical experiments. Raman measurements were used to give further experimental evidence of the formation of an adsorbed CoTSPor layer at a gold electrode by cycling the electrode potential in a CoTSPor solution. The vibrations

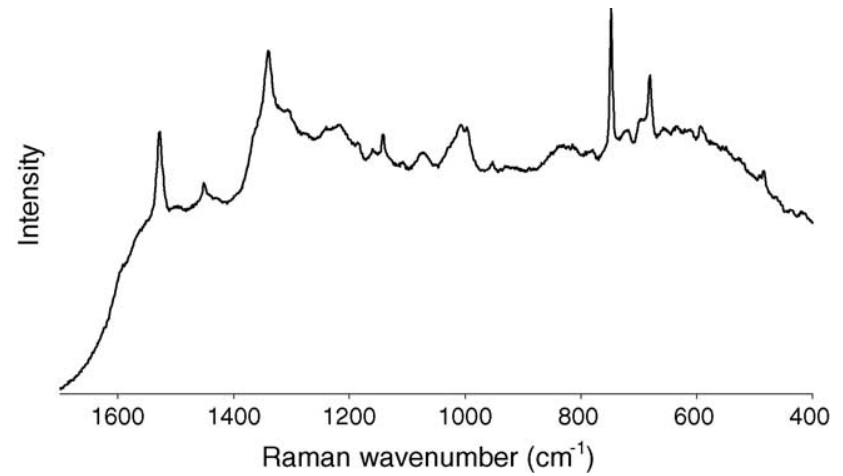

Fig. 7. Raman spectra obtained for Co(II)TSPor deposited at the surface of a gold electrode in alkaline solution.

seen in the Raman spectra are characteristic for those of a CoTSPor molecule. In addition, the Raman band positions and bandwidth are similar to those of pure crystalline substances $\left(1528,1451,748\right.$ and $\left.681 \mathrm{~cm}^{-1}\right)$ [15-16]. Spectra of the CoTSPor particles are shown in Fig. 7. When studying the modified electrodes after rinsing with $\mathrm{pH} 12$ buffer solution, it was hard to see a layer on the electrode surface, but small crystals could be found. When recording a spectrum of these grains, porphyrin spectra were obtained. These spectra were less intense than the corresponding spectra of the unrinsed electrodes. Moreover, the Raman bands, recorded from the rinsed electrodes are broader. This may be related to a lower degree of crystallinity after rinsing.

\section{Conclusion}

The hypothesis regarding the electrodeposition of CoTSPor on a gold electrode, described in this paper, is based on the electrochemical observations made while following the current-potential behaviour as a function of scan number and CoTSPor concentration. Peaks due to the Co(III)TSPor ${ }_{\text {ads }} / \mathrm{Co}$ (II)TSPor ads redox system were identified as well as peaks due to ring oxidation and reduction of CoTSPor in solution. The deposition is controlled by kinetics before the breaking point, which is the point of maximum adsorption, resulting in a chaotic multilayer of CoTSPor molecules. After the breaking point, a slow reorganisation of the chaotic multilayer occurs. The axially coordinated benzyl groups are responsible for the slowness of this process. A study of the deposited layer with spectroscopic methods, such as Raman spectroscopy, gives the ultimate proof of adsorption of the porphyrin.

\section{References}

[1] K.M. Kadish, K.M. Smith, R. Guilard, The Porphyrin Handbook, Academic Press, 2000.

[2] A.B.P. Lever, J. Porphyr. Phthalocya. 3 (1999) 488.

[3] B.Z. Nikolic, R.R. Adzic, E.B. Yeager, J. Electroanal. Chem. 103 (1979) 281. 
[4] J.H. Zagal, P. Herrera, Electrochim. Acta 30 (1985) 449.

[5] J.H. Zagal, C. Paez, Electrochim. Acta 34 (1989) 243.

[6] R. Guilard, C. Lecomte, K.M. Kadish, Struct. Bond. 64 (1987) 205.

[7] G.S. Wilson, in: A.J. Bard, M. Stratmann (Eds.), Encyclopedia of Electrochemistry, vol. 9, Wiley, Weinheim, 2002, p. 175.

[8] K. De Wael, P. Westbroek, A. Adriaens, E. Temmerman, Electroanalysis, in press.

[9] K. De Wael, P. Westbroek, E. Temmerman, J. Electroanal. Chem. 567 (2004) 167.

[10] C.C. Leznoff, A.B.P. Lever, Properties and Applications, vol. 3, VCH, Weinheim, 1993.
[11] K. Ozoemena, P. Westbroek, T. Nyokong, Electrochem. Commun. 3 (2001) 259.

[12] J. Bard, L.R. Faulkner, Electrochemical Methods Fundamentals and Applications, Wiley, New York, 2001.

[13] C.M.A. Brett, A.M.O. Brett, Electrochemistry: Principles, Methods and Applications, Oxford University Press, Oxford, 1993.

[14] R.S. Nicholson, I. Shain, Anal. Chem. 36 (1964) 706.

[15] R. Holze, Electrochim. Acta 36 (1991) 999.

[16] T.M. Cotton, S.G. Schultz, R.P. Van Duyne, J. Am. Chem. Soc. 104 (1982) 6528. 